\title{
Raman spectroscopic characterization of multiwall carbon nanotubes and of composites
}

\author{
L. Bokobza*, J. Zhang \\ Université Pierre et Marie Curie- Ecole Supérieure de Physique et Chimie Insdustrielles - Centre National de la \\ Recherche Scientifique, UMR 7615, 10 rue Vauquelin, 75231 Paris Cedex 05, France
}

Received 9 December 2011; accepted in revised form 9 February 2012

\begin{abstract}
In this work Raman spectroscopy was used for extensive characterization of multiwall carbon nanotube (MWNTs) and of MWCNTs/rubber composites. We have measured the Raman spectra of bundled and dispersed multiwall carbon nanotubes. All the Raman bands of the carbon nanotubes are seen to shift to higher wavenumbers upon debundling on account of less intertube interactions. Effects of laser irradiation were also investigated. Strong effects are observed by changing the wavelength of the laser excitation. On the other hand, at a given excitation wavelength, changes on the Raman bands are observed by changing the laser power density due to sample heating during the measurement procedure.
\end{abstract}

Keywords: polymer nanocomposites, carbon nanotubes, Raman spectroscopy

\section{Introduction}

Over the past decade, a huge scientific interest, both theoretically and experimentally, has been focused on carbon nanotubes (CNTs) due to their unprecedented properties arising from their unique onedimensional character. The combination of superb mechanical, electrical and thermal properties makes CNTs ideal reinforcing fillers for advanced composite materials. But one of the major problem is the fact that nanotubes aggregate in bundles as a result of substantial van der Waals attractions and homogeneous dispersion of CNTs in the host matrix is probably the most fundamental issue for efficient load transfer and good reinforcement [1-6].

Raman spectroscopy has been shown to be a powerful and nondestructive technique for the characterization of carbon-based materials including carbon black (CB) and carbon nanotubes (CNTs) and it has become an invaluable tool for understanding many fundamental aspects of all $\mathrm{sp}^{2}$ carbons $[7,8]$. The diameter of nanotubes [9], the presence of disorder in $\mathrm{sp}^{2}$-hybridized carbon systems [10] as well as the effect of nanotube-nanotube interactions [11] on the vibrational modes have been assessed using Raman spectroscopy. Specific features such as the strong frequency dependence on the excitation laser energy of some Raman bands [12-17] or laser radiation-induced effects [18-21] have been the subject of extensive studies.

Raman spectroscopy of CNT-based composite materials has been used to evaluate the state of dispersion and the polymer-filler interactions reflected, by shifts or width changes of the peaks. On the other hand, the sensitivity of some bands of CNTs to an application of mechanical deformation to the composite has also been used to quantify the load transferred from the matrix to the nanotubes and thus the interfacial adhesion [22-25].

This paper reports some investigations carried out by Raman spectroscopy on multiwall carbon nanotubes (MWCNTs) and on MWCNTs/rubber composites. For both materials, the dependence of Raman

\footnotetext{
${ }^{*}$ Corresponding author, e-mail: Liliane.Bokobza@espci.fr (c) BME-PT
} 
spectra on various parameters such as the excitation laser energy or heating effects induced by the laser power, will be discussed.

\section{Experimental part}

\subsection{Materials}

Multiwall carbon nanotubes (MWNTs) were purchased from Nanocyl S.A. (Belgium). In this study, we have used the Nanocyl 7000 series (purity: $90 \%$ ) produced via the catalytic carbon vapor deposition process without any further purification. Their average diameter and length are around $10 \mathrm{~nm}$ and $1.5 \mu \mathrm{m}$ respectively and their surface area between 250 and $300 \mathrm{~m}^{2} \cdot \mathrm{g}^{-1}$.

The matrix that hosts the nanotubes is an elastomer based on a styrene-butadiene rubber (SBR) (Buna VSL 5025-0 from Bayer, Germany) supplied by Formix (Orléans, France). It contains $25 \mathrm{wt} \%$ of styrene unit and a butadiene phase with cis $(10 \%)$, trans $(17 \%)$ and vinyl $(73 \%)$ configurations. It was compounded with sulfur (1.1 phr), stearic acid (1.1 phr), cyclohexylbenzothiazole sulfenamide (1.3 phr), diphenylguanidine (1.45 phr) and zinc oxide (1.82 phr), 'phr' means parts per hundred parts of rubber by weight.

\subsection{Composite processing}

An appropriate amount of MWNTs was dispersed into cyclohexane (in an approximate ratio 1:10 by weight) by sonicating the suspension for $30 \mathrm{~min}$ using a Vibra-Cell VCX 500 operating at $40 \%$ amplitude with on and off cycles respectively equal to 4 and 2 seconds.

The gum containing the SBR rubber and all the ingredients of formulation was mixed separately in cyclohexane under magnetic stirring until complete dissolution then mixed with the MWNTs dispersion. The mixture may be submitted to a further sonication for $30 \mathrm{~min}$ if global examination by optical microscopy still reveals nanotube agglomeration on a micrometer scale. The sonication process is followed by agitation under magnetic stirring until evaporation of the solvent. Total removal of any remaining solvent is achieved under vacuum overnight at $50^{\circ} \mathrm{C}$ before the cross-linking process and film formation. The unfilled and filled samples were then cured into plaques at $170^{\circ} \mathrm{C}$ during 10 min under a pressure of $150 \mathrm{bar}$ in a standard hot press. The resulting films were around $300 \mu \mathrm{m}$ thick.

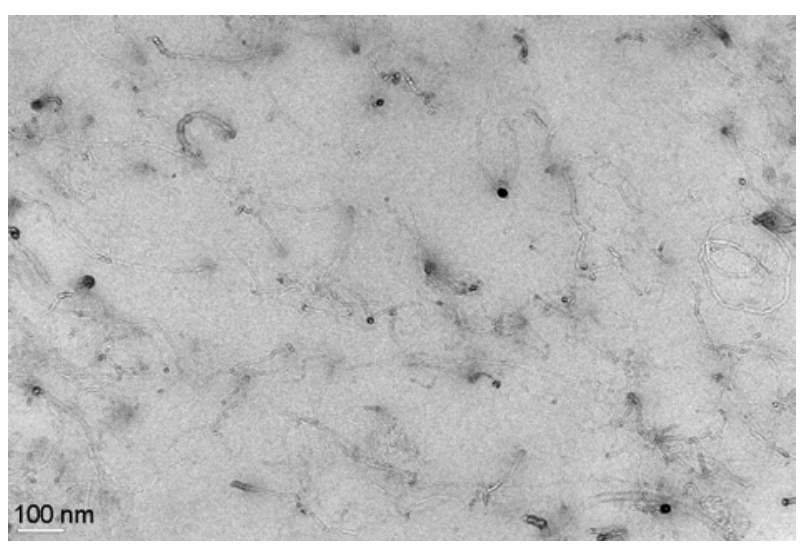

Figure 1. TEM image of the SBR-3 phr MWNTs composite

These processing conditions ensure a good dispersion of the carbon nanotubes in the elastomeric matrix as revealed by the image obtained by transmission electron microscopy (TEM) of a SBR composite filled with $3 \mathrm{phr}$ of MWNTs (Figure 1). The image was obtained from a JEOL JEM-2010 Electron Microscope (Japan), operating at $200 \mathrm{kV}$. Ultrathin sections $(50-60 \mathrm{~nm})$ were cut at $-90^{\circ} \mathrm{C}$ by using an Ultracut $\mathrm{S}$ ultramicrotome from Leica fitted with a diamond knife from Diatome.

\subsection{Raman spectra}

The Raman spectra were recorded in the backscattering geometry on a Labram HR (Jobin-Yvon, Horiba Group, France) microspectrometer in conjunction with a confocal microscope. Most of the experiments were carried out with an excitation wavelength of $514.5 \mathrm{~nm}$ from a tunable Argon laser focused on the sample by means of a $100 \times$ objective of 0.9 numerical aperture. The spot is around $3 \mu \mathrm{m}$ diameter while the beam intensity is $1 \mathrm{~mW}$. But as shown below other excitation wavelengths or laser powers were used in order to show the sensitivity of the Raman spectra to these parameters.

\section{Results and discussion \\ 3.1. Effect of tube-tube interactions on the Raman modes of MWCNTs}

The two main typical graphite bands are present in the Raman spectrum of MWCNTs bundles: the band at $1580 \mathrm{~cm}^{-1}$ ( $\mathrm{G}$ band) assigned to the in-plane vibration of the $\mathrm{C}-\mathrm{C}$ bond ( $\mathrm{G}$ band) with a shoulder around $1604 \mathrm{~cm}^{-1}$, typical of defective graphite-like materials and the band at $1342 \mathrm{~cm}^{-1}$ (D band) activated by the presence of disorder in carbon systems. The Raman spectrum also exhibits a band at 


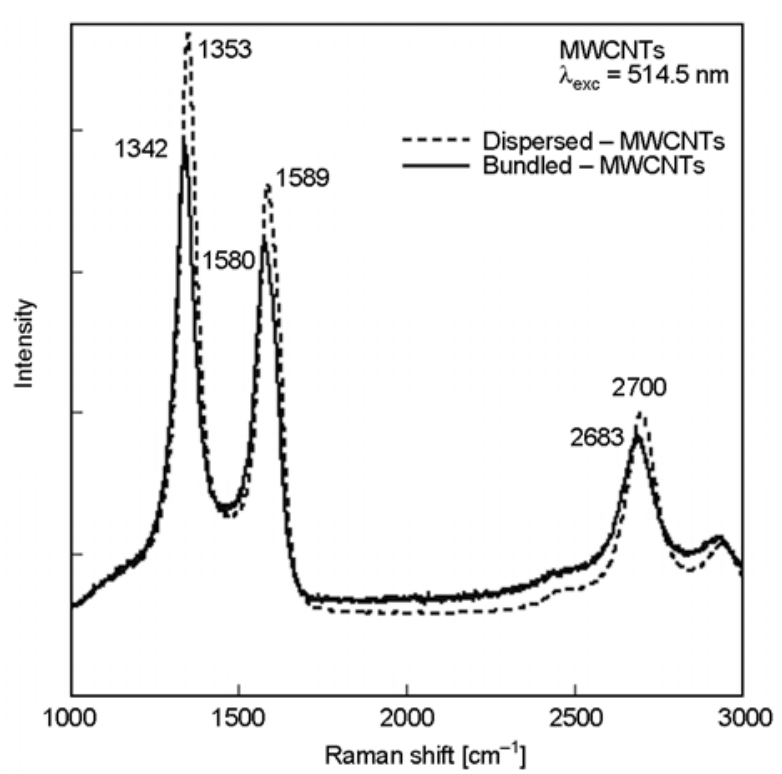

Figure 2. Raman spectra of bundled and dispersed multiwall carbon nanotubes (MWCNTs)

$2683 \mathrm{~cm}^{-1}$ called the $G^{\prime}$ band and attributed to the overtone of the $\mathrm{D}$ band. These bands have been obtained on the as-received powder of bundled MWCNTs. In Figure 2, the Raman spectrum of raw nanotubes is compared to that deposited from solution to evaluate the separation of nanotube bundles. The raw nanotubes were dispersed in cyclohexane by ultrasonication during $30 \mathrm{~min}$ and the Raman spectrum is taken after drop casting the solution on a glass slide and evaporating the organic solvent. With regard to the raw nanotubes, all the Raman bands of the dispersed nanotubes are shifted to

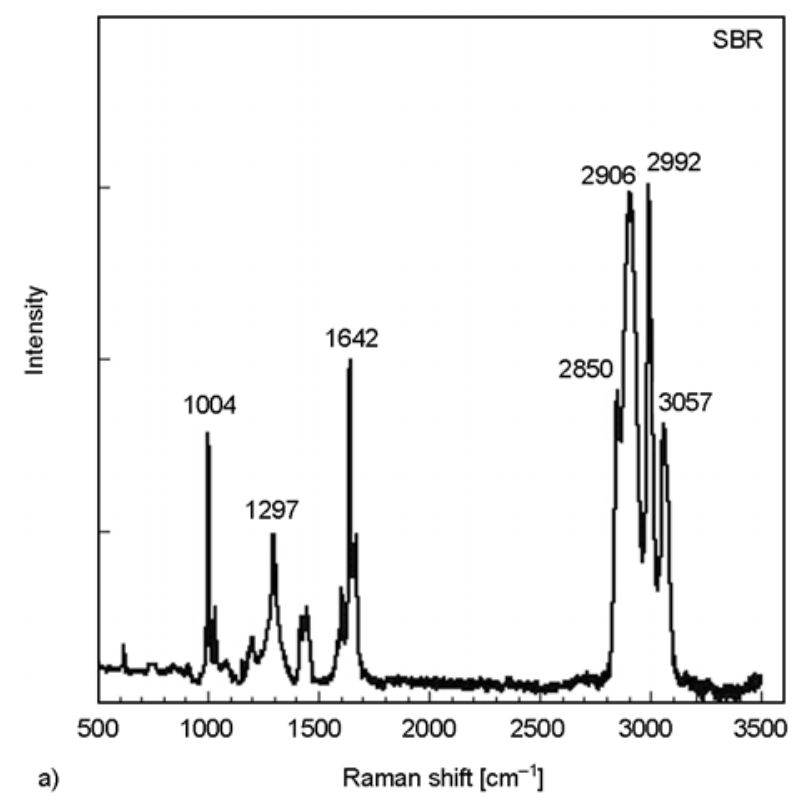

higher wavenumbers indicating less intertube interactions.

A debundling also occurs by penetration of polymer chains into nanotube aggregates during composite processing. In Figure 3 are displayed the Raman spectrum of an unfilled elastomeric matrix, it is a styrene-butadiene rubber (SBR) and that of a composite filled with $5 \mathrm{phr}$ of MWCNTs ( $\mathrm{phr}=$ parts per hundred parts of rubber by weight). With regard to the spectrum of dispersed nanotubes represented in Figure 2, a further upshifting is observed when the tubes are embedded in the host matrix as a consequence of a disentanglement and subsequent dispersion in the polymeric medium. As seen in Figure 3, the Raman spectrum of the composite is almost dominated by the bands of the nanotubes but some bands of the SBR are still present especially those located at 1005, 2856, 2917, 2995 and $3065 \mathrm{~cm}^{-1}$. These polymer bands are also shifted to higher frequencies with regard to those of the unfilled polymer as consequence of physical constraints introduced to the polymer chains by the presence of the nanotubes.

\subsection{Effect of the laser excitation energy on the Raman modes of MWCNTs}

One of the striking features of the Raman spectra of $\mathrm{sp}^{2}$ carbon materials is the linear laser excitation energy dependence of the wavenumber of the $\mathrm{D}$ and $G^{\prime}$ bands. Their strong dispersive behavior has been largely discussed in the literature [12-17] and inter-

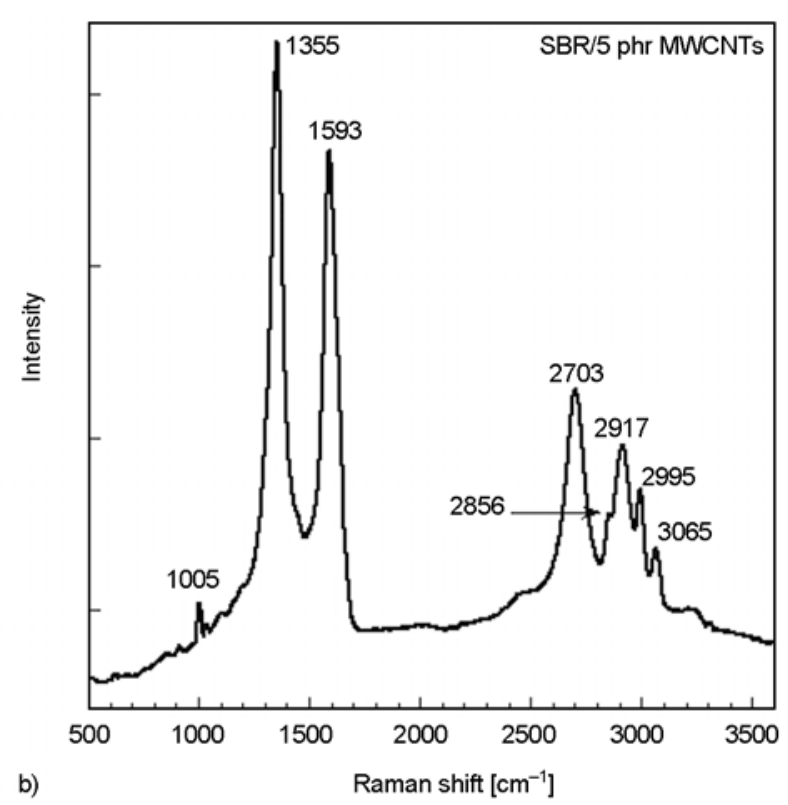

Figure 3. Raman spectra of a styrene-butadiene rubber (SBR) (a) and that of a SBR composite filled with 5 phr of MWCNTs (b) 
preted as a double resonance process involving a laser-induced resonant transition of an electron
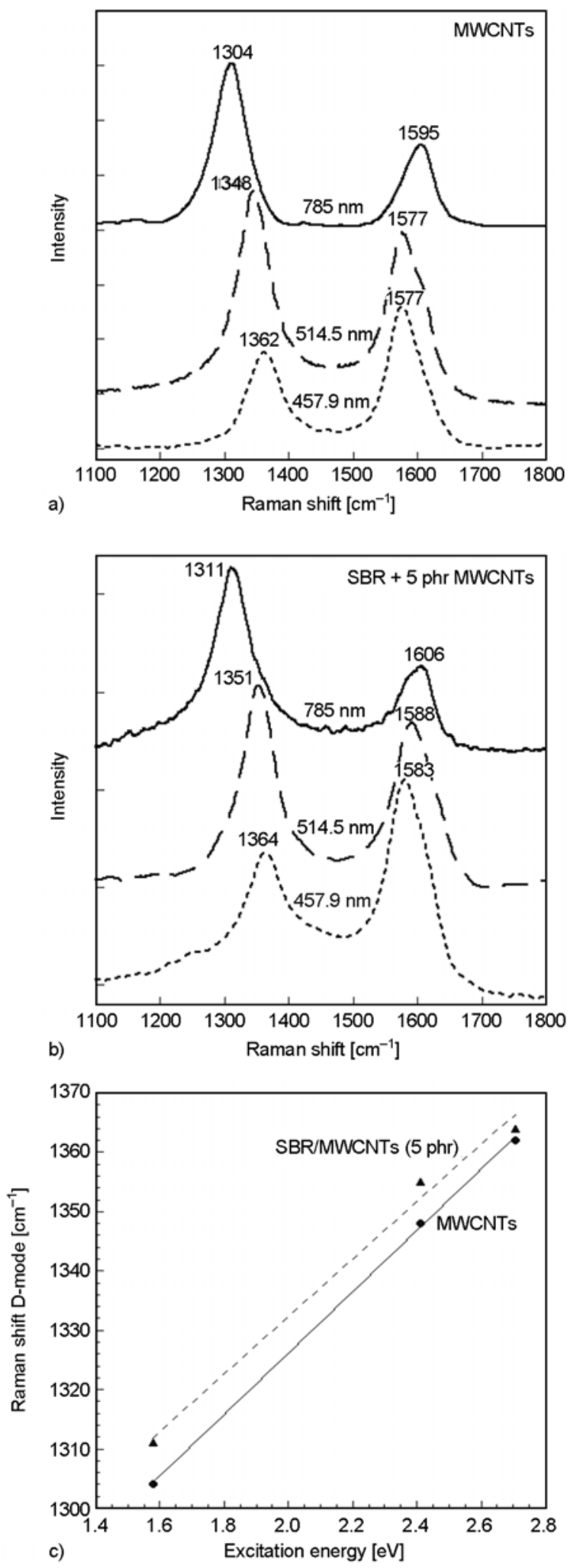

Figure 4. Raman spectra of MWCNTs (a) and of SBR$5 \mathrm{phr}$ MWCNTs composite (b) obtained with laser wavelengths $457.9,514.5$ and $785 \mathrm{~nm}$ and dependence of the wavenumber of the $\mathrm{D}$ band on laser energy (c) from the valence to the conduction band of carbon materials. A scattered resonance can take place if the electron is scattered by a phonon to a real state and after an elastic scattering process by lattice defects, the electron relaxes to the valence band [26]. Resonant Raman scattering is characterized by a strong enhancement of the detected signal when real transitions are involved. The conceptual difference between non-resonant, single-resonance and double-resonance Raman processes has been well explained by Thomsen et al. [27].

Figure 4 shows the Raman spectra of MWCNTs and of the SBR/5 phr MWCNTs composite obtained with three different laser excitation energies. For laser excitation variation, we have used the $457.9 \mathrm{~nm}(2.71 \mathrm{eV})$ and $514.5 \mathrm{~nm}(2.41 \mathrm{eV})$ lines from an Argon ion laser and the $785 \mathrm{~nm}(1.58 \mathrm{eV})$ of a NIR diode laser. As observed in single-walled carbon nanotubes (SWCNTs), the wavenumber of the $\mathrm{D}$ band increases while the ratio of the intensities $\left(I_{\mathrm{D}} / I_{\mathrm{G}}\right)$ decreases with increasing laser energy. The energy dependence of $\approx 50 \mathrm{~cm}^{-1} / \mathrm{eV}$ (Figure 4) compared well with the reported values of various authors in the literature $[13,15,17]$. Interestingly, a similar dispersive behavior is observed for the $\mathrm{D}$ band of the nanotubes in the elastomeric matrix although higher wavenumbers are obtained for each excitation energy.

\subsection{Thermal effects caused by incident laser irradiation}

Heating effects induced by the laser power of the Raman experiment have been shown to cause changes to the Raman spectrum of CNTs. Increases of the incident laser power induce corresponding increases in the temperature of CNTs resulting in the shift to lower frequency for the radial breathing mode and the G band in SWCNTs [18] and for all the bands in MWCNTs [28]. Li et al. [18] suggest that the ability to induce the temperature increase in carbonaceous materials originates from the presence of impurities, defects and disorder in the samples. On the other hand, it has also been shown that electrical heating induce downshifts in the Raman frequencies, basically identical to those obtained by laser illumination thus indicating that the change in the Raman spectrum originates from laser-induced heating effect. Zhang et al. [19] show that under intense laser irradiation, the $G$ band frequency of 
unpurified SWCNTs undergoes reversible variation indicating that the tubes were not damaged in the irradiation process. In addition, the integrated $\mathrm{D}$ and $\mathrm{G}$ bands intensities ratio $\left(I_{\mathrm{D}} / I_{\mathrm{G}}\right)$ decreases rapidly in the initial increase in laser power but remains basically unchanged in the subsequent increase in laser power indicating a lowering in the degree of disorder. The authors conclude that intense laser irradiation can lead to the loss of impurities and results in a purified CNT sample. In a study by Raman spectroscopy on the stability of carbon nanotubes to laser irradiation, Olevik et al. [29] mention the particular sensitivity of CNT bundle materials to overheating due to inefficient cooling in this system compared to invividual CNTs and show that irreversible destruction of CNTs in the bundles can occur at even low laser power density $\left(0.15 \mathrm{~kW} / \mathrm{cm}^{2}\right)$. From the above reported results, it appears that laser induced effects are very important to consider for the analysis of the Raman spectrum of CNTs. Heating the sample by the incident laser radiation may cause reversible changes but the original spectrum may not be recovered upon a decrease of the laser power owing to a damage of the tubes. The frequency downshifts induced by the local increase of the sample temperature due to laser irradiation have been associated with a lengthening of the $\mathrm{C}-\mathrm{C}$ bond resulting from the thermal expansion of the material [30]. Nevertheless, changes in resonance conditions, softening of the intra-tubular van der
Waals interactions or thermal expansion of the bundles are other reasons invoked to account for the dependence of Raman spectra on increasing temperatures [20,31].

We report here a study of the influence of laser irradiation on the Raman spectra of MWCNTs and of MWCNTs/SBR composites. We have used five laser power levels in this study corresponding to 1,10 , 25,50 and $100 \%$ of the highest laser power which is $10 \mathrm{~mW}$. In order to check if laser irradiation causes reversible effects, the spectra were first taken at the lowest laser power $(1 \%)$ then the laser power was gradually increased to $100 \%$ then decreased to $1 \%$ again.

The Raman spectra of MWCNTs irradiated with various laser powers at $514.5 \mathrm{~nm}$ are shown in Figure 5. The overall Raman signal increases with the laser power but as shown in Figure 6, downshifts of both $\mathrm{D}$ and $\mathrm{G}$ frequencies are observed with increased laser power. The spectral variations induced by laser heating are reversible, which means that the nanotubes are not damaged by the irradiation process. A difference in the peak position is obtained for the lowest laser power on account of the poor Raman signal and less accuracy in the determination of the wavenumbers.

A thermogravimetric analysis performed on MWCNTs from room temperature to $800^{\circ} \mathrm{C}$ leaves a residue that displays a Raman spectrum quite similar to that observed in Figure 1 with D and G bands respec-
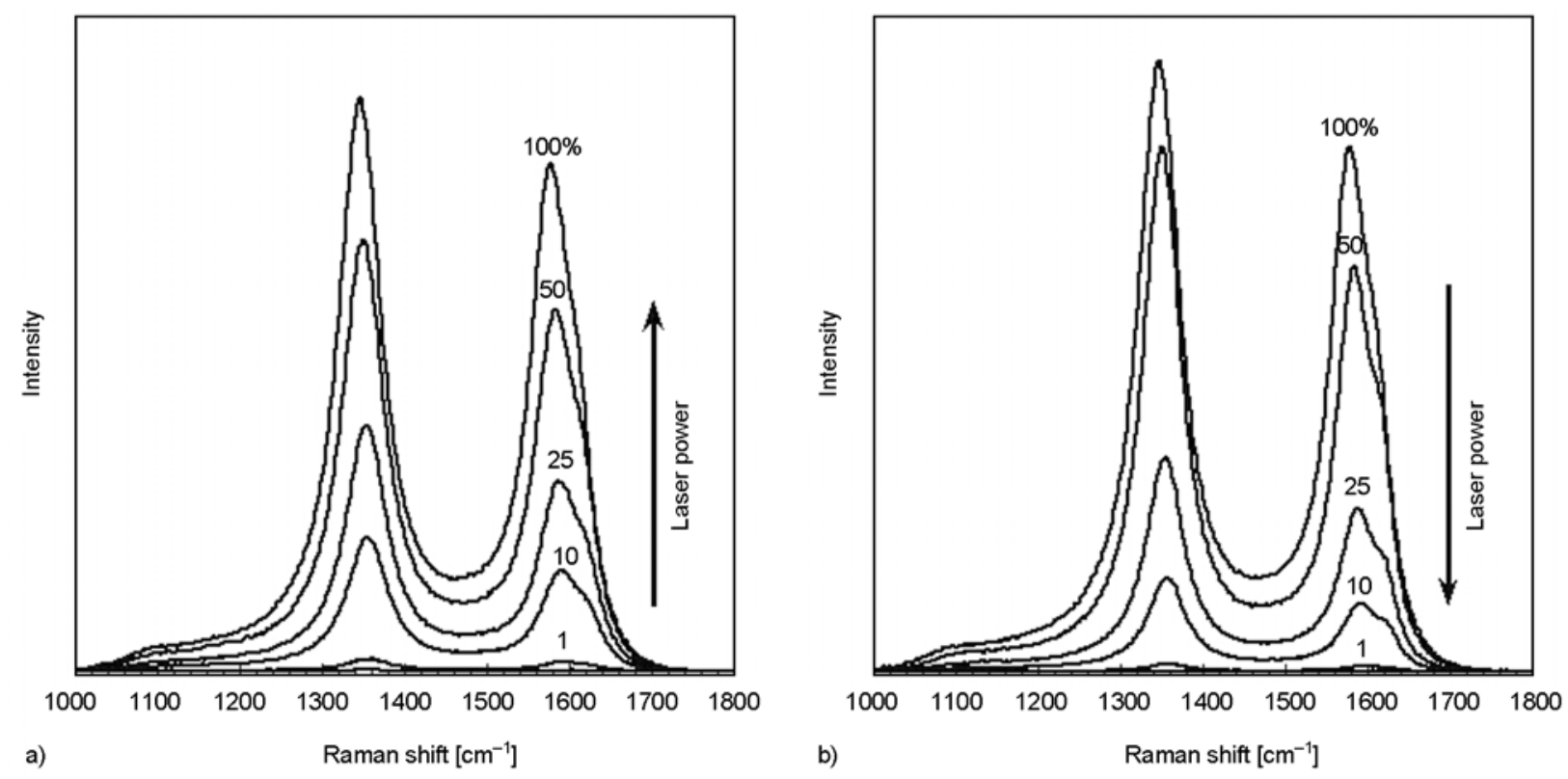

Figure 5. Raman spectra of MWCNTs samples irradiated at $514 \mathrm{~nm}$ taken at increasing laser power from 1 to $100 \% \cdot 10 \mathrm{~mW}$ (a) and decreasing laser power from 100 to $1 \% \cdot 10 \mathrm{~mW}$ (b) 

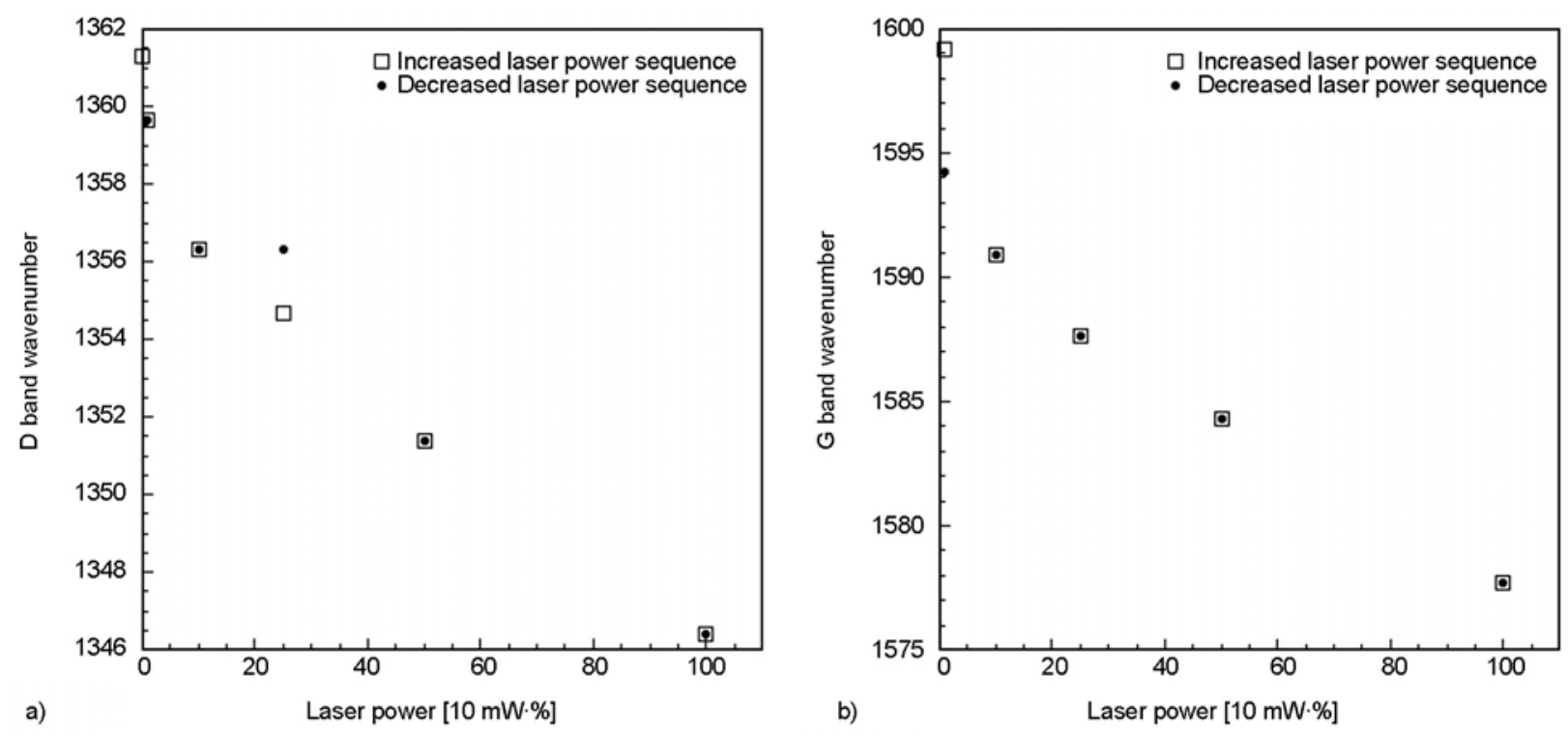

Figure 6. Laser power dependences of the D (a) and G (b) bands of MWCNTs

tively located at 1351 and $1588 \mathrm{~cm}^{-1}$. This result shows that heating the nanotubes does not destroy them which confirms the spectroscopic analysis if, of course, Raman changes under laser irradiation are induced by heating effects.

Raman spectra were also taken at the same spot on SBR/MWCNTs composites for different laser power densities going from low to higher power densities. But for each sample, several experiments at different spots were performed in order to confirm the results. Features of interest are the positions of the $\mathrm{D}$ and $\mathrm{G}$ bands that downshift, as for non-embedded MWCNTs (Figure 6), with increasing laser power

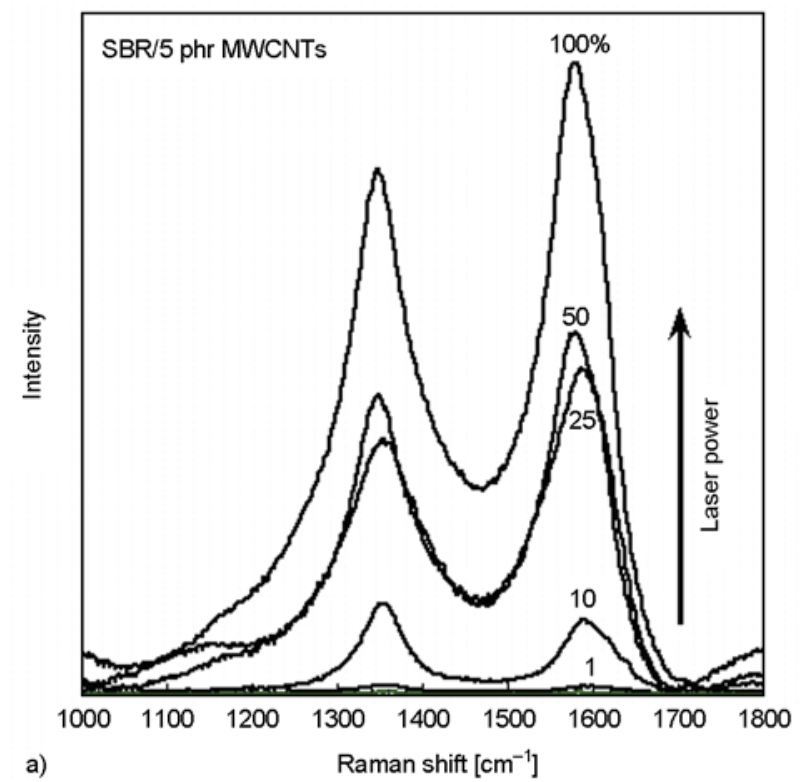

and the laser-induced frequency shifts are reversible. More surprising is the fact that above a given laser power $(10 \mathrm{~mW} \cdot 25 \%$ in the spectra of the SBR $/ 5 \mathrm{phr}$ MWCNTs represented Figure 7a), the intensity of the $\mathrm{D}$ band becomes lower than that of the $\mathrm{G}$ band. The $D$ band remains less intense than the $G$ band upon further increase of the laser power and also after progressive decrease of the laser power from the highest value (Figure 7b). This can be visualized by looking at the dependence of the $I_{\mathrm{D}} / I_{\mathrm{G}}$ ratio on the laser power represented in Figure $8 . I_{\mathrm{D}} / I_{\mathrm{G}}$ decreases rapidly with increasing laser power but remains almost unchanged in the subsequent

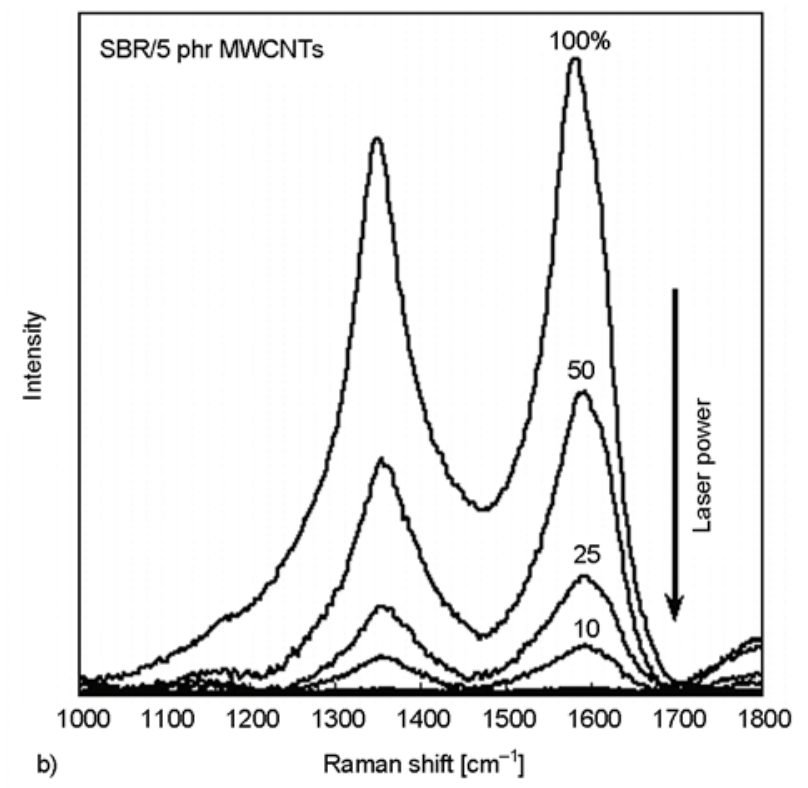

Figure 7. Raman spectra of the SBR/5 phr MWCNTs composite irradiated at $514.5 \mathrm{~nm}$ taken at increasing (a) and decreasing (b) laser power [10 $\mathrm{mW} \% \%]$ 


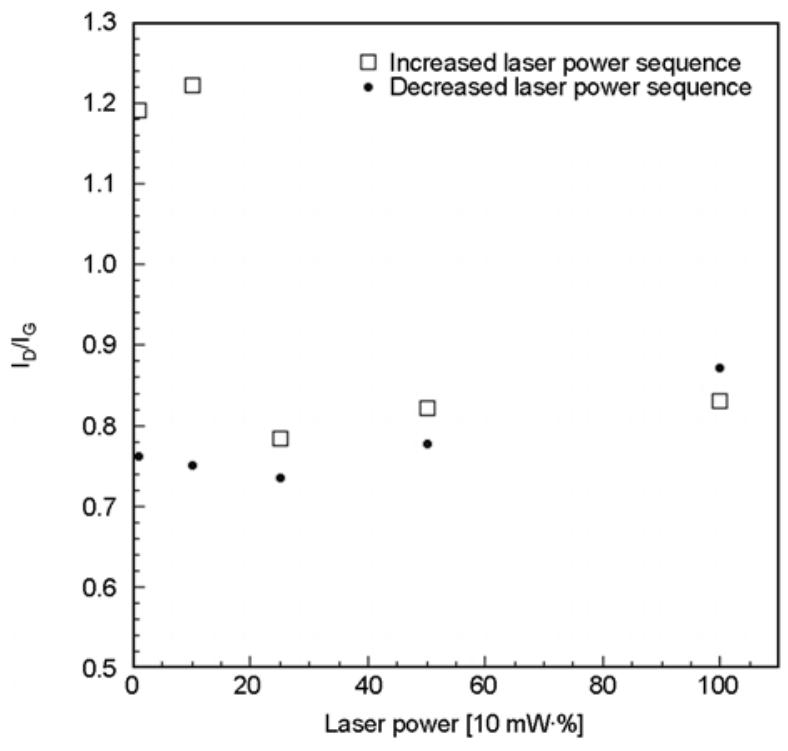

Figure 8. Dependence of the $I_{\mathrm{D}} / I_{\mathrm{G}}$ ratio of the SBR/5 phr MWCNTs composite on the laser power

increase. In contrast, $I_{\mathrm{D}} / I_{\mathrm{G}}$ is almost unchanged in the decreasing laser power cycle. This result is quite similar to that obtained by Zhang et al. [19] in a study devoted to the effects of intense laser irradiation on Raman intensity features of SWCNTs. As the $I_{\mathrm{D}} / I_{\mathrm{G}}$ ratio reflects the degree of disorder, the authors conclude that the irreversible change in intensity resulting from intense laser irradiation originates from sample purification. But why the change in intensity of the D band only occurs in the MWCNT composites and not in non-embedded MWCNTs?

We think that overheating effects due to laser irradiation are probably more important in the composites on account of the low thermal conductivity of the surrounding matrix. Despite the high thermal conductivity of individual nanotubes - around $6000 \mathrm{~W} /(\mathrm{m} \cdot \mathrm{K})$ for SWCNTs) and around $3000 \mathrm{~W} /(\mathrm{m} \cdot \mathrm{K})$ for MWCNTs [32], the SBR matrix can be considered as a thermal insulator since its thermal conductivity, around $0.120 \mathrm{~W} /(\mathrm{m} \cdot \mathrm{K})$, is only enhanced by $70 \%$ by addition of $10 \mathrm{phr}$ of MWCNTs. So the heat generated by light illumination is not dissipated resulting in high temperature increase of nanotube and purification.

\section{Conclusions}

In this work, Raman spectroscopy has proved to be a convenient and useful method for carbon nanotube characterization. Nevertheless local increase of the sample temperature due to laser irradiation may cause irreversible changes in the Raman spectra making difficult the correct spectra acquisition and interpretation.

\section{References}

[1] Andrews R., Weisenberger M. C.: Carbon nanotube polymer composites. Current Opinion in Solid State and Materials Science, 8, 31-37 (2004).

DOI: $10.1016 /$ j.cossms.2003.10.006

[2] Hu Y., Shenderova O. A., Hu Z., Padgett C. W., Brenner D. W.: Carbon nanostructures for advanced composites. Reports on Progress in Physics, 69, 18471895 (2006).

DOI: $10.1088 / 0034-4885 / 69 / 6 / \mathrm{R} 05$

[3] Coleman J. N., Khan U., Blau W. J., Gun'ko Y. K.: Small but strong: A review of the mechanical properties of carbon nanotube-polymer composites. Carbon, 44, 1624-1652 (2006). DOI: $10.1016 /$ j.carbon.2006.02.038

[4] Moniruzzaman M., Winey K. I.: Polymer nanocomposites containing carbon nanotubes. Macromolecules, 39, 5194-5205 (2006). DOI: $10.1021 / \mathrm{ma} 060733 \mathrm{p}$

[5] Bokobza L.: Multiwall carbon nanotube elastomeric composites: A review. Polymer, 48, 4907-4920 (2007). DOI: $10.1016 /$ j.polymer.2007.06.046

[6] Spitalsky Z., Tasis D., Papagelis K., Galiotis C.: Carbon nanotube-polymer composites: Chemistry, processing, mechanical and electrical properties. Progress in Polymer Science, 35, 357-401 (2010).

DOI: 10.1016/j.progpolymsci.2009.09.003

[7] Dresselhaus M. S., Jorio A., Hofmann M., Dresselhaus G., Saito R.: Perspectives on carbon nanotubes and graphene Raman spectroscopy. Nano Letters, 10, 751758 (2010).

DOI: $10.1021 / \mathrm{n} 1904286 \mathrm{r}$

[8] Jorio A., Pimenta M. A., Souza Filho A. G., Saito R., Dresselhaus G., Dresselhaus M. S.: Characterizing carbon nanotube samples with resonance Raman scattering. New Journal of Physics, 5, 139/1-139/17 (2003). DOI: $10.1088 / 1367-2630 / 5 / 1 / 139$

[9] Graupner R.: Raman spectroscopy of covalently functionalized single-wall carbon nanotubes. Journal of Raman Spectroscopy, 38, 673-683 (2007). DOI: $10.1002 /$ jrs. 1694

[10] Cuesta A., Dhamelincourt P., Laureyns J., MartínezAlonso A., Tascón J. M. D.: Raman microprobe studies on carbon materials. Carbon, 32, 1523-1532 (1994). DOI: 10.1016/0008-6223(94)90148-1

[11] Rao A. M., Chen J., Richter E., Schlecht U., Eklund P. C., Haddon R. C., Venkateswaran U. D., Kwon Y-K., Tománek D.: Effect of van der Waals interactions on the Raman modes in single walled carbon nanotubes. Physical Review Letters, 86, 3895-3898 (2001). DOI: $10.1103 /$ PhysRevLett.86.3895 
[12] Pócsik I., Hundhausen M., Koós M., Ley L.: Origin of the $\mathrm{D}$ peak in the Raman spectrum of microcrystalline graphite. Journal of Non-Crystalline Solids, 227-230, 1083-1086 (1998).

DOI: 10.1016/S0022-3093(98)00349-4

[13] Matthews M. J., Pimenta M. A., Dresselhaus G., Dresselhaus M. S., Endo M.: Origin of dispersive effects of the Raman $D$ band in carbon materials. Physical Review B, 59, R6585-R6588 (1999).

DOI: 10.1103/PhysRevB.59.R6585

[14] Thomsen C., Reich S.: Double resonant Raman scattering in graphite. Physical Review Letters, 85, 52145217 (2000).

DOI: $10.1103 /$ PhysRevLett.85.5214

[15] Brown S. D. M., Jorio A., Dresselhaus M. S., Dresselhaus G.: Observations of the $D$-band feature in the Raman spectra of carbon nanotubes. Physical Review B, 64, 073403/1-073403/4 (2001).

DOI: 10.1103/PhysRevB.64.073403

[16] Sood A. K., Gupta R., Asher S. A.: Origin of the unusual dependence of Raman $D$ band on excitation wavelength in graphite-like materials. Journal of Applied Physics, 90, 4494-4497 (2001).

DOI: $10.1063 / 1.1408590$

[17] Kürti J., Zólyomi V., Grüneis A., Kuzmany H.: Double resonant Raman phenomena enhanced by van Hove singularities in single-wall carbon nanotubes. Physical Review B, 65, 165433/1-165433/9 (2002). DOI: $10.1103 /$ PhysRevB.65.165433

[18] Li H. D., Yue K. T., Lian Z. L., Zhan Y., Zhou L. X., Zhang S. L., Shi Z. J., Gu Z. N., Liu B. B., Yang R. S., Yang H. B., Zou G. T., Zhang Y., Iijima S.: Temperature dependence of the Raman spectra of single-wall carbon nanotubes. Applied Physics Letters, 76, 2053 2055 (2000).

DOI: $10.1063 / 1.126252$

[19] Zhang L., Li H., Yue K-T., Zhang S-L., Wu X., Zi J., Shi Z., Gu Z.: Effects of intense laser irradiation on Raman intensity features of carbon nanotubes. Physical Review B, 65, 073401/1-073401/4 (2002).

DOI: $10.1103 /$ PhysRevB.65.073401

[20] Zhou Z., Dou X., Ci L., Song L., Liu D., Gao Y., Wang J., Liu L., Zhou W., Xie S., Wan D.: Temperature dependence of the Raman spectra of individual carbon nanotubes. Journal of Physical Chemistry B, 110, 1206-1209 (2006). DOI: $10.1021 / j p 053268 \mathrm{r}$

[21] Zhang Y., Son H., Zhang J., Kong J., Liu Z.: Laserheating effect on Raman spectra of individual suspended single-walled carbon nanotubes. Journal of Physical Chemistry C, 111, 1988-1992 (2007). DOI: 10.1021/jp066016e

[22] Zhao Q., Wagner H. D.: Raman spectroscopy of carbon-nanotube-based composites. Philosophical Transactions of the Royal Society A: Mathematical, Physical and Engineering Sciences, 362, 2407-2424 (2004). DOI: $10.1098 /$ rsta.2004.1447
[23] Kao C. C., Young R. J.: A Raman spectroscopic investigation of heating effects and the deformation behaviour of epoxy/SWNT composites. Composites Science and Technology, 64, 2291-2295 (2004).

DOI: 10.1016/j.compscitech.2004.01.019

[24] Kannan P., Eichhorn S. J., Young R. J.: Deformation of isolated single-wall carbon nanotubes in electrospun polymer nanofibres. Nanotechnology, 18, 235707/1235707/7 (2007).

DOI: $10.1088 / 0957-4484 / 18 / 23 / 235707$

[25] Mu M., Osswald S., Gogotsi Y., Winey K. I.: An in situ Raman spectroscopy study of stress transfer between carbon nanotubes and polymer. Nanotechnology, 20, 335703/1-335703/7 (2009).

DOI: $10.1088 / 0957-4484 / 20 / 33 / 335703$

[26] Reich S., Thomsen C.: Raman spectroscopy of graphite. Philosophical Transactions of the Royal Society A: Mathematical, Physical and Engineering Sciences, 362, 2271-2288 (2004).

DOI: $10.1098 /$ rsta.2004.1454

[27] Thomsen C., Reich S., Maultzsch J.: Resonant Raman spectroscopy of nanotubes. Philosophical Transactions of the Royal Society A: Mathematical, Physical and Engineering Sciences, 362, 2337-2359 (2004). DOI: 10.1098/rsta.2004.1444

[28] Huang F., Yue K. T., Tan P., Zhang S-L., Shi Z., Zhou X., Gu Z.: Temperature dependence of the Raman spectra of carbon nanotubes. Journal of Applied Physics, 84, 4022-4024 (1998). DOI: $10.1063 / 1.368585$

[29] Olevik D., Soldatov A. V., Dossot M., Vigolo B., Humbert B., McRae E.: Stability of carbon nanotubes to laser irradiation probed by Raman spectroscopy. Physica Status Solidi B, 245, 2212-2215 (2008). DOI: $10.1002 /$ pssb.200879661

[30] Huong P. V., Cavagnat R., Ajayan P. M., Stephan O.: Temperature-dependent vibrational spectra of carbon nanotubes. Physical Review B, 51, 10048-10051 (1995). DOI: 10.1103/PhysRevB.51.10048

[31] Meletov K. P., Krestinin A. V., Arvanitidis J., Christofilos D., Kourouklis G. A.: Temperature effects in the Raman spectra of bundled single-wall carbon nanotubes. Chemical Physics Letters, 477, 336-339 (2009). DOI: $10.1016 /$ j.cplett.2009.07.001

[32] Hone J., Whitney M., Piskoti C., Zettl A.: Thermal conductivity of single-walled carbon nanotubes. Physical Review B, 59, R2514-R2516 (1999).

DOI: 10.1103/PhysRevB.59.R2514 